\title{
ACADÉMICOS CONTRA LA POBREZA: UNA IDEA PARA LA QUE HA LLEGADO SU TIEMPO ${ }^{1}$
}

\section{Academics Stand Against Poverty: An Idea WhOSE Time Has COME}

\author{
THOMAs POGGE y LUIS CABRERA ${ }^{2}$ \\ Yale University (Estados Unidos) - University of Birmingham (Inglaterra)
}

\author{
RECIBIDO: 9 DE NOVIEMBRE DE 2011 \\ ACEPTADO: 19 DE DICIEMBRE DE 2011
}

\begin{abstract}
Resumen: El último cuarto de siglo ha sido desastroso para el mundo pobre. Entre 1988 y 2005, las familias que pertenecen a la cuarta parte más pobre de la humanidad han perdido un tercio de sus ingresos, lo que supone que su participación se reduce al $0,78 \%$ de los ingresos de toda la humanidad.

Desmintiendo muchos informes sobre la pobreza, que tiñen los datos más positivamente ${ }^{3}$ y aun a pesar de los compromisos [de los países ricos] ${ }^{4}$, repetidamente publicitados, como los Objetivos del Desarrollo del Milenio (ODM), encontramos que el número de personas con desnutrición crónica se ha incrementado alcanzando los mil millones por vez primera en la historia de la humanidad. Las muertes debidas a la pobreza se calculan en torno a los dieciocho millones anualmente e implican una tercera parte de todos los decesos. La necesidad de mejorar tales índices no admite excusas. ¿Hay
\end{abstract}

\footnotetext{
${ }^{1}$ Traductor: José Barrientos Rastrojo. Agradecemos las correcciones realizadas a la traducción por David Álvarez.

${ }^{2}$ Thomas Pogge es Leitner Professor de Filosofía y Asuntos Internacionales en la Yale University. Luis Cabrera es Profesor Titular [Reader] en Teoría Política en la University of Birmingham.

${ }^{3}$ Cfr. CHANDY, L. - GERTZ, G.: “Quantifying Poverty's Global Decline," Brookings Institution, octubre 2011, disponible on-line en www.brookings.edu/articles/ 2011/10_poverty_global_decline_chandy.aspx. Chandy y Gertz afirman que la pobreza global, medida de acuerdo con la línea internacional de la pobreza del Banco Mundial de 1,25 dólares diarios en Paridad de Poder Adquisitivo, ha mostrado una constante tendencia a la baja debido especialmente al crecimiento de China y de otras potencias emergentes. Una discusión acerca de algunos serios problemas relativos a la medición de la pobreza del Banco Mundial, así como cuestiones similatrs relativas a la meta de los ODM de reducir la pobreza global para 2015, puede consultase en los capítulos 3 y 4 de POGGE, Th.: Politics As Usual: What Lies Behind the Pro-Poor Rhetoric, Cambridge, Polity, 2010 [trad. castellana en Thomas Pogge; Hacer justicia a la humanidad. México, F.C.E. 2009, cap 7 y en Paulette Dieterlen (comp.) Los derechos económicos y sociales. Unamirada desde la filosofía. Máxico, UNAM cap. 7]. Véanse también las páginas 100-107 para encontrar razones para el escepticismo respecto a aquellos que afirman el crecimiento y la reducción de la pobreza en China. [pp. 292-305 en Hacer justicia a la humanidad].

${ }^{4}$ Nota del traductor: Hemos añadido algunos anexos entre corchetes a lo largo del texto para facilitar su comprensión.
} 
algo que nosotros como académicos podamos y debamos hacer para amparar a los que se encuentran en esa posición?

En este ensayo discutimos algunas contribuciones específicas que investigadores y profesores pueden llevar a cabo. El argumento se dirige especialmente a aquellos cuyo trabajo se centra en aspectos como la pobreza, pero entendemos que los profesores de universidad e investigadores de todas las disciplinas pueden realizar una contribución específica desde su rol como académicos. Comenzamos con algunos comentarios sobre las razones acerca de por qué los académicos se deberían sentir compelidos, en términos prácticos y políticos, a comprometer más directamente sus esfuerzos en la erradicación de la pobreza más aguda. Luego, ofreceremos ejemplos más específicos de tales implicaciones, incluyendo algunos proyectos de intervención que ya existen. Responderemos a las críticas que objetan, por una parte, que esos "ingenuos bienhechores" no deberían implicarse en debates o que, por otra, aseveran que a los académicos se les exige demasiado en su trabajo o que, por último, los deberes relativos a la pobreza respecto a los compatriotas deberían tener prioridad sobre las necesidades de pobreza absoluta de la gente que situados en cualquier parte del mapa [sin interesar si pertenecen o no a nuestro país]. Las preocupaciones procedentes de cada crítica, sostenemos, resultan menos convincentes que las ganancias obtenidas de un mayor nivel de compromiso. Cerramos [nuestra exposición], comentando la existencia de una asociación internacional fundada recientemente, Academics Stand Against Poverty (ASAP) [Académicos Contra la Pobreza], que desea ayudar a los académicos a comprometerse en los senderos detallados en este ensayo con el fin de erradicar la pobreza más grave instalada en todo el planeta. ASAP también proporciona un medio para que los académicos que ya están trasladando sus ideas a un público más amplio (asesorando agencias gubernamentales o internacionales de ayuda o, quizás, a ONGs o a corporaciones internacionales) compartan sus valiosas habilidades y conocimientos con otros.

Palabras clave: académicos, pobreza, ética

Abstract: The last quarter century has been disastrous for the world's poor. Between 1988 and 2005 ,
the poorest quarter of humanity lost a third of its share of global household income, seeing this share
shrink to a grotesque 0.78 percent. Giving the lie to many rosy poverty reports, ${ }^{5}$ and despite highly
publicized commitments such as the Millennium Development Goals (MDGs), the number of
chronically undernourished people has steadily increased, breaking above 1 billion for the first time
in human history. Deaths from poverty-related causes still number around 18 million annually,
accounting for about one-third of all human deaths. The need to do better is overwhelmingly obvious.
Is there anything we as academics can and should do to help protect the world's poor?
In this essay, we discuss some specific contributions that researchers and teachers can make. The
argument is mainly addressed to those whose work focuses on aspects of poverty, but we understand
that scholars from all disciplines can make distinct contributions in their roles as academics. We

${ }^{5}$ Véase, por ejemplo, Laurence Chandy y Geoffrey Gertz, "Quantifying Poverty’s Global Decline," Brookings Institution, October 2011. www.brookings.edu/articles/2011/ 10_poverty_global_decline_chandy.aspx Chandy y Gertz arguyen que la pobreza mundial, tal y como es medida por el Banco Mundial que establece el límite de la misma en el gasto de 1,25 dólares diarios en paridad con el poder adquisitivo con el primer mundo, han mostrado una tendencia a la baja en los últimos años, especialmente debido al crecimiento de China y de otros países de poder emergente. Una discusión de algunos problemas graves de los instrumentos de medida usado por el Banco Mundial y de otros asuntos de medida relacionados con el intento de reducir a la mitad la pobreza mundial en 2015 según los ODM puede consultarse en los capítulos 3 y 4 de POGGE, T.: Politics As Usual: What Lies Behind the Pro-Poor Rhetoric, Cambridge, Polity, 2010. Véanse también las páginas 100-107 acerca de la razones para ser escépticos sobre las aseveraciones que aseguran el crecimiento y la reducción de la pobreza en China. 
begin with some general remarks on reasons why academics should feel compelled to become more directly engaged - in both practical and political terms - in efforts to eradicate severe poverty. We then offer some more specific examples of such engagement, including some existing intervention projects. We respond to critics who object that 'naive do-gooders' should not insert themselves into debates, that too much may be demanded of individual academics, or that duties to relatively poor compatriots should take priority over the needs of absolutely poor people elsewhere. The concerns raised by each criticism, we argue, are less compelling than the gains that could be realized through more direct engagement. We close by discussing a recently formed international association, Academics Stand Against Poverty (ASAP), which seeks to help academics engage in the ways detailed in this essay with the goal of eradicating severe poverty worldwide. ASAP also provides a means for those academics already taking their ideas to broader public audiences — advising government aid agencies or NGOs, perhaps, or corporations or international agencies - to share their valuable skills and expertise with others.

Keywords: Academics, poverty, ethics.

\section{¿Por qué hay que tomar partido?}

Muchas personas dentro y fuera de la academia creen que es inapropiado para los académicos participar en debates públicos de forma partidista: apoyar u oponerse a tratados específicos o a secciones específicas de la legislación vigente, criticar o defender determinadas figuras o agencias políticas o decisiones. Los académicos deberían presentar los resultados de sus investigaciones -hechos, teorías, argumentaciones-, asumir el discurso político y permitir a sus participantes [a los políticos] relacionarse con sus estudios científicos como les plazca. Manteniendo cierta distancia de los acalorados debates políticos actuales, la academia conserva su dignidad y reputación gracias a que se protege su objetividad o, al menos, eso dice la teoría ${ }^{6}$.

Atisbamos algún mérito en este argumento. Sin embargo, consideramos que, tal y como el mundo se encuentra, hay razones más fuertes en la otra dirección; particularmente, cuatro. Primero, los asuntos políticos que encaran los representantes públicos y la ciudadanía en general son de enorme relevancia por lo que, si los académicos pueden ocuparse de ellos de un modo concertado, las ganancias obtenidas tendrán mucho más peso que cualquier pérdida de dignidad y reputación académica. Segundo, los académicos en las sociedades modernas detentan un cargo público que implica ciertas expectativas y deberes relacionados con un compromiso

\footnotetext{
${ }^{6}$ Para una articulación representativa de ese argumento, véanse los filósofos de la ética normativa (cfr. GAUS, G.: "Should Philosophers Apply Ethics?” Think, Primavera 2005, pp. 63-67).
} 
[social]. Tercero, muchos académicos ya se han implicado en debates públicos, con frecuencia pagados por organizaciones que poseen intereses en relación a los resultados obtenidos. En estos casos la dignidad de la academia ya se encuentra comprometida y el silencio sólo cederá el terreno a los académicos de alquiler. Finalmente, a través de su formación y rol social, muchos académicos están adecuadamente preparados para ayudar a aliviar la pobreza a través de importantes contribuciones tales como amplificar la voz del pobre.

\section{La urgencia de las cuestiones}

La humanidad enfrenta problemas ecológicos potencialmente catastróficos, incluyendo el descomunal cambio climático ${ }^{7}$ y el agotamiento de recursos naturales relevantes y no renovables como el petróleo. Encaramos la proliferación de tecnologías extremadamente peligrosas, incluyendo las armas nucleares y biológicas, que podrían diezmar la especie humana ${ }^{8}$. Además, la humanidad sufre una catástrofe silenciosa de pobreza severa que supone la tercera parte de la mortandad mundial y causa sufrimiento inimaginable por hambrunas, enfermedades y otras privaciones 9 .

Después de que los gobiernos mundiales prometiesen en la Cumbre Mundial de Alimentación de Roma de 1996 disminuir a la mitad el

\footnotetext{
${ }^{7}$ El cambio climático, por ejemplo, podría generar efectos graves sobre la producción global de alimentos, incrementando dramáticamente el hambre en muchos países pobres (Cfr. BROWN, M.E FUNK C.C.: "Food Security Under Climate Change," Science 319, n. 5863, febrero 2008, pp. 58081; LOBELL, D. - BURKE, M. B. - TEBALDI, C. - MASTRANDREA, M.D. - FALCON, W.P. NAYLOR, R.L.: "Prioritizing Climate Change Adaptation Needs for Food Security in 2030," Science 319, $\mathrm{n}^{\circ}$. 5863, febrero 2008, pp. 607-10. Véase también el trabajo de Simon Caney citado más adelante.

${ }^{8}$ Cfr. CIRINCIONE, J.: Bomb Scare: On The History and Future of Nuclear Weapons, New York, Columbia University Press, 2007, especialmente el capítulo 5. Cirincione explica cómo, aunque las tensiones nucleares no sean tan seguras como en el periodo más álgido de la Guerra Fría, aun existen cuestiones urgentes por las que preocuparse.

${ }^{9}$ La información detallada sobre la pobreza global se ha abierto al público en los últimos años, sin embargo se mantienen algunos de los problemas metodológicos de medición citados más arriba. Puede consultarse la discusión que, aunque se apoya en algunas mediciones problemáticas, ofrece no obstante, una cartografía geográfica instructiva de la pobreza global. Ver ELVIDGE, C.D. SUTTON, P.C. - GHOSH, T. - TUTTLE, B. T. - BAUGH, K.E. - BHADURI, B. BRIGHT, E.: "A Global Poverty Map Derived from Satellite Data", Computers \& Geosciences 35, $\mathrm{n}^{\circ}$ 8, 2009, pp. 1652-60.
} 
número de personas desnutridas crónicas para el 2015, esta cifra de hecho se incrementó - durante una década de caída de precios de los alimentos - desde 788 a 843 millones. Este incremento experimentó una aceleración en 2006, cuando los precios de la comida empezaron a subir. Con los precios de los alimentos en niveles máximos este año (www.fao.org), es probable que el número de personas desnutridas crónicas en 2011 alcance otro record histórico por encima de la marca de los mil millones.

La explicación simple para este fenómeno es el rápido crecimiento de la desigualdad global. Durante el periodo entre 1988 y 2005, la cuarta parte más pobre de la población humana vio reducir sus ingresos anuales en cerca de un tercio, llegando a pasar de unos ingresos de $1,16 \%$ y a $0,78 \%$ respecto a los ingresos totales mundiales. La participación de la mitad más pobre se redujo de un 3,53\% a un 2,92\% . Como consecuencia de una marginación económica tan rápida, las personas pobres no pueden ejercer suficiente demanda en el mercado para inducir a los granjeros a plantar los alimentos básicos que necesitan en lugar de los cultivos usados para producir biodiesel que compran las poblaciones más ricas.

Las cifran ponen de manifiesto que la pobreza severa es hoy evitable. La mayor parte de la que existe no lo haría si los pobres hubieran participado proporcionalmente en el crecimiento económico global reciente. De hecho, los frutos de este crecimiento se han focalizado en el cinco por ciento superior de la población mundial que ha aumentado su participación en la renta doméstica mundial del 42,87 al 46,36\% en el periodo 1988-2005.

Una explicación obvia de por qué le ha ido tan bien a los más ricos del planeta en las últimas décadas es que ellos, especialmente los más ricos de todos, disponían de las mejores oportunidades para influir, a través de sus gobiernos, en la arquitectura institucional supranacional emergente que ha sido consagrada, y rodeada, por el Tratado de la Organización Mundial del Comercio (OMC). Sin duda, los ricos no odian a los pobres, pero sus esfuerzos para influir en las normas supranacionales y para aplicarlas son, como era de esperar, guiadas por sus propios intereses económicos y políticos. Los arreglos institucionales supranacionales poseen claramente la huella de esos intereses. Por ejemplo:

1.Los países ricos y sus empresas compran enormes cantidades de 
materias primas a los gobernantes de los estados en desarrollo sin importarles cómo esas autoridades han alcanzado o ejercen su poder. En muchos casos, esto conlleva la colaboración en el robo de esos recursos a sus propietarios, los ciudadanos de la nación. Esta práctica enriquece a sus opresores $\mathrm{y}$, consecuentemente, afianza la opresión: los tiranos venden los recursos naturales de sus víctimas y posteriormente emplean los beneficios para comprar las armas que necesitan para mantenerse en el poder ${ }^{10}$.

2.Los estados ricos y sus bancos prestan dinero a esos gobernantes y obligan a la gente del país a devolverlo incluso cuando el tirano se ha marchado. Muchas poblaciones pobres están todavía pagando las deudas contraídas por cleptócratas como Suharto en Indonesia, Mobutu en la República Democrática del Congo y Abacha en Nigeria. Tal conjunto de préstamos y el consiguiente cobro de las deudas equivale a un robo: la imposición unilateral de las cargas de la deuda sobre las poblaciones empobrecidas.

3. Los países ricos favorecen la malversación de fondos por parte de los cargos públicos de los países menos desarrollados, al permitir que sus bancos acepten estos fondos. Esta complicidad se puede evitar fácilmente: a los bancos se les solicitan ya informes relacionados con fondos sospechosos de estar vinculados con el terrorismo o con el tráfico de drogas. Sin embargo, los bancos occidentales todavía aceptan y manejan con avidez fondos producto de la malversación, asegurándose de que sus bancos siguen siendo atractivos para este tipo de depósitos ilícitos. Global Financial Integrity (GFI) estima que los países menos desarrollados han perdido con esta estrategia entre 342 y 404,7 mil millones de dólares anualmente en el periodo 2000-2008.Los estados ricos y sus bancos prestan dinero a esos gobernantes y obligan a la gente del país a devolverlo incluso cuando el tirano se ha marchado. Muchas poblaciones pobres están todavía pagando las deudas provocadas por cleptócratas como Suharto en Indonesia, Mobutu en la República Democrática del Congo y Abacha en Nigeria. Tal conjunto de préstamos y

\footnotetext{
${ }^{10}$ Cfr. POGGE, T.: World Poverty and Human Rights: Cosmopolitian Responsibilities and Reforms, Cambridge, Polity Press, 2008 (segunda edición) y WENAR, L.: "Property Rights and the Resource Curse", Philosophy and Public Affairs 36, n 1, 2008, pp. 2-32.
} 
consiguientes cobro de deudas anima al robo: la imposición unilateral de deudas lastra las poblaciones empobrecidas ${ }^{11}$.

4. Los países ricos facilitan la evasión de impuestos en los países menos desarrollados a través de normas de contabilidad más laxas para las empresas multinacionales. Puesto que no han de presentar informes país a país, pueden manipular fácilmente los precios de transferencia entre sus subsidiarias para concentrar los beneficios donde se pagan menos impuestos. Como resultado, señalan que no obtuvieron beneficios en los países donde extrajeron, manufacturaron y vendieron sus bienes y servicios y pagan los impuestos de sus beneficios mundiales en algún paraíso fiscal del planeta donde sólo poseen una presencia legal pero no efectiva. El GFI estima que durante el periodo 2002-2006, la manipulación de los precios comerciales privó a las naciones menos desarrolladas de 98,4 mil millones en recaudación fiscal por año ${ }^{12}$.

5. Los países ricos generan cuotas de polución global desproporcionadas. Sus emisiones son las principales causantes de serios riesgos para la salud, fenómenos meteorológicos extremos, elevación de los niveles del mar y al cambio climático, a los que las poblaciones pobres son especialmente vulnerables. Un informe reciente del Foro Humanitario Global (Global Humanitarian Forum) destacó que el cambio climático afecta ya seriamente a 325 millones de personas, causa anualmente pérdidas económicas por valor de 125 mil millones de dólares, así como 300 mil muertes de las que el $99 \%$ se producen en los países menos desarrollados ${ }^{13}$.

6. Los países ricos han creado un régimen global de mercado que, se suponía, liberaría grandes ganancias colectivas a través de los mercados libres y abiertos. Pero el régimen está trucado: permite a

\footnotetext{
${ }^{11}$ Cfr. KAR, D. - CURCIO, K.: Illicit Financial Flows from Developing Countries: 2000-2009, Washington, Global Financial Integrity, 2011. Estas pérdidas son cuatro veces más grandes que toda la ayuda oficial para el desarrollo, que durante este periodo alcanzó una media de ochenta y tres millones de dólares anuales, de los que sólo ocho millones se dedicaron a "los servicios sociales más básicos" (Fuente: Indicadores de los ODM de las Naciones Unidas consultadas en http://unstats.un.org/unsd/mdg/Search.aspx?q=bss\%20oda, último acceso 16 de diciembre de 2011).

${ }^{12}$ Cfr. HOLlinSHEAD, A.: The Implied Tax Revenue Loss from Trade Mispricing, Washington, Global Financial Integrity, 2010, p. 15, Tabla 2.

${ }^{13}$ Cfr. GLOBAL HUMANITARIAN FORUM: The Anatomy of a Silent Crisis, Geneva, Global Humanitarian Forum, 2009, pp. 1 y 60-61.
} 
los estados ricos continuar protegiendo sus mercados a través de tarifas y regulaciones "antidumping" para alcanzar mayores cuotas de mercado gracias a créditos y subsidios para la exportación (incluyendo los, aproximadamente, 227 mil millones de dólares anuales aportados sólo en agricultura) que los países pobres no puede darse el lujo de igualar ${ }^{14}$. Puesto que la producción, en los países pobres, requiere más mano de obra que en los desarrollados, tales medidas proteccionistas destruyen muchos más puestos de trabajos de los que crean.

Estos puntos nos traen más razones para explicar por qué es tan urgente que el tema de la pobreza sea tratado por los académicos. Tanto los académicos como sus estudiantes y lectores suelen pertenecer a los países más ricos y, probablemente, se encontrarán entre los beneficiarios de los acuerdos institucionales internacionales, que basculan a favor de ellos mismos. Así, probablemente, ostentemos responsabilidades especiales para explorar y destacar las injusticias estructurales que nuestros gobiernos diseñan y defienden en nuestro nombre ${ }^{15}$.

\section{El cargo académico}

La segunda razón por la que los académicos deben tomar partido es que ellos ostentan un cargo público en las sociedades modernas. Este cargo implica ciertas expectativas y responsabilidades. Cuando se realizan debates públicos que giran sobre asuntos que requieren pericia académica, el público espera que los académicos contribuyan con sus conocimientos. Cuando se crea un debate público importante, por

\footnotetext{
${ }^{14}$ Cfr. Organization for Economic Cooperation and Development (OECD): Agricultural Policy Monitoring and Evaluation 2011: OECD Countries and Emerging Economies, Paris, OECD, 2011, p. 18 (también señala que en 2010 los subsidios del gobierno representaron el dieciocho por ciento de los beneficios de las granjas pertenecientes a los países de la OECD).

${ }^{15}$ Estas responsabilidades especiales se pueden fundar en derechos negativos, donde se entiende que los individuos contribuyen a los daños identificados y así están obligados a ayudarlos a remediarlos. Una fundamentación complementaria basada en deberes positivos consideraría que los que disfrutan de mayor seguridad material en los estados ricos se encuentran en una posición adecuada para defender los cambios en aquellos aspectos del gobierno que se ocuparían de estos daños. Tendrían por ello el deber positivo de llevarlo a cabo. Para un estudio que enfatiza el primer enfoque, ver los capítulos cuatro a seis del libro citado de Thomas Pogge World Poverty and Human Rights. Véase también Cabrera, L.: The Practice of Global Citizenship, Cambridge, Cambridge University Press, 2010, pp. 90-95.
} 
ejemplo sobre si son necesarios los esfuerzos internacionales para reducir la emisión de gases con efecto invernadero, se espera que los científicos contribuyan con el conocimiento probado del que dispongan.

Dada esta esperanza, el silencio académico se puede interpretar, razonablemente, como la aceptación de estos profesionales de que las principales perspectivas representadas en el debate público son creíbles y consistentes con los datos disponibles. Aquellos que aceptan los cargos académicos, que implican estas expectativas, tienen la responsabilidad de vivir conforme a esta premisa, tanto como aquel que acepta el cargo de vigilante de la playa y posee la responsabilidad de rescatar a bañistas en peligro en la orilla del mar. Si los especialistas en el clima no señalan que los datos disponibles apoyan la hipótesis de un cambio climático causado por el hombre [antropogenica], será razonable pensar que el juicio todavía no se ha decidido, es decir, que una hipótesis alternativa permanece como un candidato factible. En ese caso, serán responsables de los efectos de sus palabras: la demora consiguiente en tomar urgentemente acciones necesarias para reducir la emisión de gases de efecto invernadero.

Este deber se extiende plausiblemente a la formación en las clases. $\mathrm{Ni}$ que decir tiene que los profesores académicos no han de adoctrinar a sus estudiantes con ninguna perspectiva específica sobre la pobreza global. Por el contrario, en tanto que configuradores de temas específicos y subcampos disciplinares, deben asegurar que ofrecen un compendio de información amplio y crítico a los estudiantes que asumen - o deberían asumir - aspectos de pobreza global en su formación. Un curso de Introducción a las Relaciones Internacionales puede, por ejemplo, incorporar una unidad o contenido trasversal dedicado a la pobreza y a los posibles deberes transfronterizos de resolverla. Excluir este tema, da lugar a pensar que no es un asunto de importancia para el estudio de la política mundial. Una reflexión análoga se puede hacer sobre un amplio espectro de los cursos de las ciencias sociales y las humanidades, así como de muchas ciencias naturales y la medicina, donde las implicaciones en torno a asuntos relacionados con la pobreza pueden destacarse aun en estudiantes de los primeros cursos. Dada la urgencia de los asuntos identificados arriba, es plausible exigir que el docente de la 
clase posea la responsabilidad de incorporar los datos más destacados sobre la pobreza en los temarios de las diversas asignaturas.

El deber de dar repuesta

Íntimamente relacionado con la idea del académico como aquel que ostenta un cargo destacado, se ubica la comprensión de que son responsables de dar respuestas cuando, en su ámbito de conocimientos, se hagan públicas falsas afirmaciones, especialmente por otros académicos. De hecho, la exhortación actual de que deben permanecer fuera de los debates públicos manifiesta una gran dosis de ingenuidad. Cuando es mucho lo que está en juego, los académicos pueden conseguir elevadas recompensas apoyando a una de las posiciones. El hecho de que los académicos son sensibles a tales incentivos puede observarse en el sistema judicial de Estados Unidos, donde muchos ganan beneficios lucrativos por proporcionar, de modo predecible, un testimonio de peso a quienquiera que los contrate para testificar ${ }^{16}$. También, en los debates públicos encontramos muchos académicos sucumbiendo a tales recompensas y, por lo tanto, apoyando al lado que más dinero ofrece, con frecuencia el lado incorrecto. Las guerras de recompensas sobre los daños producidos por el tabaco muestran un ejemplo excelente de este fenómeno: durante varias décadas contrataron a expertos académicos para prolongar la impresión de que los datos sobre sus efectos no eran concluyentes ${ }^{17}$. Análogamente, expertos en farmacia han aceptado los pagos de grandes corporaciones para pregonar la seguridad y eficacia de medicinas muy caras. Incluso hay muchos académicos, becados con frecuencia por multinacionales interesadas, que dispuestos a negar la realidad del cambio climático con causa humana [antropogénica].

Estos fenómenos son, tal vez, más aterradores en el debate sobre los efectos de nuestras instituciones emergentes de gobernanza global sobre la evolución de la pobreza. Las corporaciones se juegan billones en que la percepción pública de que los drásticos cambios institucionales que

\footnotetext{
${ }^{16}$ Véase una discusión sobre asuntos destacados sobre el uso de testigos expertos, en particular economistas en el contexto estadounidense en POSNER, R.A.: "The Law and Economics of the Economic Expert Witness," Journal of Economic Perspectives 13, no 2, Primavera, 1999, pp. 91-99.

${ }^{17}$ Véase una discusión sobre asuntos destacados sobre el uso de testigos expertos, en particular economistas en el contexto estadounidense en POSNER, R.A.: "The Law and Economics of the Economic Expert Witness,” Journal of Economic Perspectives 13, nº 2, Primavera, 1999, pp. 91-99.
} 
lograron a través de la presión que ejercen son buenos para todos, incluidos los pobres. Mientras ellos, sus políticos y expertos propagan el mito persuasivo de que la marea que sube levanta igualmente a todos los barcos, los más necesitados, que no disponen de expertos en publicidad bien pagados a los que contratar para que cuenten su versión de los hechos, son la mayor parte de los casos silenciados. El resultado es un mundo singular en el que casi todos los expertos disponibles públicamente están de acuerdo en que el estilo de globalización predominante, bajos los auspicios de la OMC, constituye una gran oportunidad para el mundo de los más desfavorecidos, aun cuando el número de los desnutridos crónicamente alcanza casi todos los años nuevos records. En este País de las Maravillas, nuestro mundo actual, no queda una puridad académica que pueda ser preservada: el silencio de los expertos universitarios reforzará la percepción pública de que la globalización de la $\mathrm{OMC}$ ha sido positiva para los que tienen menos. El público atribuirá, con razón, este punto de vista a los expertos que permanecen callados. Así pues, legítimamente supondrán que si los informes emitidos por los gobiernos y sus organizaciones internacionales fueran falsos o tendenciosos, hubieran sido cuestionados por muchos de estos expertos universitarios.

Si los expertos examinasen visiblemente e impugnasen estos informes, el público y los medios de comunicación desarrollarían una actitud más crítica y podría más atención al problema de la pobreza. Este examen más cuidadoso, además, provocaría que los defensores del status quo que han sido gratificados económicamente para afirmar su posición realizasen aseveraciones con más miramiento y más atención a los datos, puesto que encararían un riesgo real de bochorno público (un riesgo que actualmente es despreciable). No es realista pensar que todos los impedimentos académicos para la erradicación de la pobreza absoluta puedan ser eliminados. No obstante, como académicos, podemos y debemos contribuir a reducir tales impedimentos allá donde sea posible llevarlo a término fácilmente.

\section{Las capacidades de los académicos}


Por ultimo, los investigadores y profesores preocupados por la pobreza poseen deberes específicos para comprometerse fundados en su potencial para realizar contribuciones significativas, distintivas y complementarias a otros empeños, tales como aquellos implicados en las campañas desarrolladas por grandes $\mathrm{ONG}^{18}$. Tales académicos recibieron años de formación intensiva en contenidos y metodologías. Su conocimiento esencial con frecuencia excede mucho de los que crean las políticas sociales, los periodistas y aquellos que se encargan normalmente de presentar las cuestiones que son de relevancia para combatir la pobreza. Que este conocimiento superior y experto, si contribuye efectivamente a los debates públicos y a los procesos de creación de políticas, podría tener un impacto trascendental resultando fuertemente precisado es algo que se nos sugiere por la magnitud y severidad de las privaciones y dificultades sufridas que afectan a la pobreza global.

Cuando la agresión de Alemania y Japón amenazaron la civilización humana, muchos académicos cambiaron radicalmente sus actividades para contribuir con su trabajo experto a la meta de derrotar a las potencias del eje. Hoy, aprobamos y celebramos tales esfuerzos ${ }^{19}$. Sin embargo, muchos creen que los tiempos actuales son diferentes: normales, pacíficos y benignos. De hecho, así podría parecer desde un punto de vista ventajoso y privilegiado situado en uno de los países ricos. Sin embargo, la pobreza causa actualmente más muertes y sufrimiento que las provocadas por la Segunda Guerra Mundial durante sus años más $\operatorname{oscuros}^{20}$. Y las catástrofes del cambio climático podrían infligir sobre

\footnotetext{
${ }^{18}$ Un ejemplo sería la campaña de Oxfam GROW, que persigue indagar en los problemas fundamentales de la producción y la distribución de alimentos. Puede consultarse en: www.oxfam.org.uk/get_involved/system/, último acceso 1 de diciembre de 2011.

${ }^{19}$ Cfr. MASS, A. - HOIJMAIJERS, H. (eds.): Scientific Research in World War II: What Scientists Did During the War, Abingdon, Routledge, 2009. Ni que decir tiene que algunos esfuerzos, como el desarrollo de armas nucleares, simplemente no se pueden aplaudir. En el contexto de este ensayo es importante subrayar que muchos de los científicos cuyo trabajo era instrumental en el desarrollo de esas armas ayudaron después de la guerra a controlar su difusión. Einstein en particular suspendió el foco de su trabajo científico para realizar campañas para una solución política global. Consúltese CAUDILL, S.: “"Trying to Harness Atomic Energy, 1946-1951: Albert Einstein's Publicity Campaign for World Government," Journalism Quarterly 1-2, Primavera/Verano 1991, pp. 253-62.

${ }^{20}$ El número total de muertes relacionadas con la Segunda Guerra Mundial (1939-1945) se estima que fue superior a los cuarenta y ocho millones: entre ocho y diez millones por año. La cifra incluye siete millones y seiscientas mil muertes de militares y más de tres millones de civiles y catorce millones y doscientas mil personas entre los militares de los aliados y más de veinticuatro millones
} 
nuestros descendientes un impacto aun más horrendo que la peor guerra de la historia de la humanidad. La necesidad de actuar no permite excusas y se exige de forma inmediata. Aquellos economistas, científicos del medio ambiente, especialistas en estudios del desarrollo, científicos políticos, filósofos y otros con capacidades sobresalientes en los problemas de la pobreza global pueden y deberían sentirse obligados a poner sus competencias tan altamente desarrolladas al servicio de la arena pública. Los que ya están inmersos en el diálogo y la consulta pública de los gobiernos y los agentes del desarrollo pueden aumentar su impacto mediante la coordinación personal con otros sujetos que piensan del mismo modo dentro de la academia.

\section{Aquello que los académicos pueden hacer y están realizando}

Por tanto, ¿qué pueden y deben hacer los académicos, concretamente en relación a estas finalidades?, ¿cómo podemos cumplir con nuestras responsabilidades en relación al público y el mundo de la pobreza?, ¿Cómo podrían nuestros talentos y experiencias únicos realizar una contribución específica para cumplir el gran deber moral de la humanidad de acabar con la pobreza sevra evitable tan pronto como sea posible? Proponemos aquí un marco de tres elementos para realizar esta contribución. Implica:

1) alcanzar audiencias más amplias,

2) lograr un impacto sobre la pobreza a través de intervenciones más directas e

3) incentivar la inclusión de las voces de los pobres del mundo.

La asunción que subyace a cada una es, repitámoslo, que los profesores e investigadores comprometidos en tales esfuerzos pueden incrementar su impacto por medio de una participación colaborativa en un grupo tal como Académicos contra la pobreza [Academics Stand Against Poverty] (www.academicsstand.org), al que nos referiremos más abajo.

En primer lugar, los académicos procedentes de varios campos pueden comprometerse en extender publicamente su mensaje, lo que en el 
argot de las agencias de financiación de la investigación se denomina "transferencia del conocimiento". Es posible compartir nuestro conocimento de expertos sobre los asuntos relativos a la pobreza específica a través de medios escritos, medios telemáticos, medios televisivos, debates públicos, testimonios ante órganos de gobierno y colaboraciones con corporaciones y organizaciones de la vida social. Tales actividades pueden ser cruciales para presentar nuevos descubrimientos, cuestionar las asunciones del discurso público y especialmente para ayudar a encuadrar el discurso de la pobreza mundial con por aportaciones apropiadas de la academia.

Por ejemplo, siguiendo nuestras posiciones del inicio, los académicos poseerían un rol crucial para evaluar críticamente las noticias demasiado optimistas que difunden muchos gobiernos y organizaciones intergubernamentales. Por medio de esta acción, podemos perfilar y ampliar las demandas populares para conseguir una acción más poderosa.

Espacios como el "Poverty Matters Blog" del periódico The Guardian sobre asuntos de desarrollo global proporciona una plataforma pública para compartir ideas y presentar desafíos a las políticas y acciones de diversas agencias ${ }^{21}$.

Hay dos tareas más relacionadas íntimamente con este punto. Al dirigir más empeños a explorar las causas de la persistencia de la pobreza, podemos estimular a los políticos y a los ciudadanos a elevar demandas más específicas que las meras descripciones de lo que está sucediendo (tal como aparece en los ODM) y así conseguir formular peticiones que actores singulares deban llevar a término ${ }^{22}$. Articulando con claridad las bases del imperativo para la erradicación de la pobreza, es posible hacer que sea más difícil que sea excluido de las agendas políticas nacionales e internacionales.

\footnotetext{
${ }^{21}$ La blog se encuentra suscrita financieramente por la Fundación Bill y Melinda Gates. Su dirección online es www.guardian.co.uk/global-development/poverty-matters

${ }^{22}$ Para conseguir más información acerca de la falta de voluntad de los estados para hacer firme y dentro de tiempo los compromisos alcanzados en los ODMs y que sólo han cumplido leves matizaciones de los mismos, consúltese HULME, D.: "The Millennium Development Goals (MDGs): A Short History of the World's Biggest Promise", Brooks World Poverty Institute Working Paper 100 (September 2009), pp. 36-43, disponible online en: www.bwpi.manchester.ac.uk/resources/Working-Papers/bwpi-wp-10009.pdf, último acceso 1 de diciembre de 2011.
} 
La segunda parte del modelo apunta al impacto, es decir, a los esfuerzos para contribuir más directamente a la disminución de la pobreza. Esto también es una tarea en la que ponen cada vez más énfasis las agencias de financiación situadas en Inglaterra, Europa y otros lugares. En Inglaterra, donde una porción significativa de los fondos públicos para la universidad se decide en función de una revisión profunda de la investigación, que se lleva a cabo aproximadamente cada seis años, todos los departamentos se espera que discutan actualmente el impacto tangible de sus actividades de investigación. El impacto en este contexto trasciende la mera transferencia de información al gobierno o a la masa. Por el contrario, implica efectos demostrables y concretos en la política gubernamental, en los esfuerzos de la ONGs o en las vidas de personas reales. Este criterio podría resultar muy elevado para aquellas contribuciones académicas que no sea cuantificables de modo directo e inmediato. Por supuesto, aceptamos justificables las críticas de un instrumentalismo económico a esta posición que promueve exclusivamente el impacto y la transferencia de conocimiento ${ }^{23}$. A pesar de ello, el concepto de impacto puede ser integrado adecuadamente. En el contexto de la pobreza global, miles de académicos de todo el mundo son capaces de realizar contribuciones directas y potencialmente significativas: individual o colectivamente $\mathrm{y}$, con frecuencia, asociados a agentes no académicos.

En términos de actividades conjuntas, por ejemplo, una iniciativa temprana de ASAP ha ayudado a reunir voces universitarias dentro del discurso global emergente sobre los objetivos que deberían reemplazar a los ODM, que expiran en $2015^{24}$. Este proyecto ofrecerá un complemento destacado para una campaña a cargo de la coalición de ONGs denominada "Beyond 2015", la cual pretende construir "un movimiento global, plural y que plenamente inclusivo [multi-stakeholder] para construir un marco legítimo post-2015" ${ }^{25}$. Ambos esfuerzos se pueden inteligir como unas contribuciones importantes para el diálogo sobre la

\footnotetext{
${ }^{23}$ Cfr. SWAIN, H.: "Higher Education White Paper is Provoking a Winter of Discontent," The Guardian, 27/09/2011.

${ }^{24}$ El proyecto se titular "Informe sobre el consenso de la pobreza global. Puede ampliarse la información en www.academicsstand.org, último acceso 1 de diciembre de 2011.

${ }^{25}$ La website de Beyond 2015 es http://beyond2015.org/, último acceso 1 de diciembre de 2011.
} 
implementación y efectividad de los ODM, que ha implicado a un gran número de académicos trabajando con agencias de las Naciones Unidas y de otras instituciones ${ }^{26}$. Estos esfuerzos más recientes pueden interpretarse como la continuación del diálogo y como un medio para aportar cuestionamientos y correcciones [a lo ya realizado].

Otro proyecto pretende evaluar sistemáticamente la efectividad de las organizaciones que luchan contra la pobreza con el objetivo de canalizar las ayudas económicas allí donde manifiesten mejores resultados. Este proyecto interdisciplinario y basado en GiveWell $^{27}$-una iniciativa de la sociedad civil, conforma, en sí mismo, un complemento destacado para las nacientes investigaciones académicas sobre los resultados de la ayudas, de su efectividad y sobre sus responsabilidades ${ }^{28}$. Por otra parte, GiveWell proporciona un modelo y oportunidades para los académicos de verse más directamente implicados.

\footnotetext{
${ }^{26}$ El proyecto del milenio de las Naciones Unidas fue liderado por el economista Jeffrew Sachs. Durante su dirección del comisionado de las Naciones Unidas en el periodo 2002-2006, realizó su trabajo desde las recomendaciones de un número amplio de expertos acerca de cómo implementar y alcanzar las Principales Metas de Desarrollo del Milenio. Esto incluía reducir a la mitad el hambre crónica global, conseguir educación primaria para todos los niños, disminuir la mortalidad infantil y maternal, reducir las muertes por tuberculosis, por SIDA y por malaria. Los informes finales están disponibles en www.unmillenniumproject.org (último acceso 1 de diciembre de 2011). Los directores de los proyectos difundieron activamente las ideas, incluyendo los numerosos informes con opiniones evaluativas y apoyando el esfuerzo general de consecución de los ODMs a través de su exposición en los principales periódicos. Para una crítica representativa del diálogo público generado acerca del esfuerzo para alcanzar los ODMs, específicamente sobre algunos desafíos que generar los instrumentos de medida en relación con las metas esperadas, véase la editorial realizada por invitación por el colaborador inicial de Sachs y director en la fundación de Global Fund to Fight AIDS, Tuberculosis and Malaria, Amir Attaran "Necessary Measures," New York Times, 13/09/2005, disponible online en www.nytimes.com/2005/09/13/opinion/13attaran.html, último acceso 1 de diciembre de 2011. Véase también ATTARAN, A.: "An Immeasurable Crisis? A Criticism of the Millennium Development Goals and Why They Cannot Be Measured," PloS Medicine 2, $\mathrm{n}^{\circ} 10$, 25/09/2005.

${ }^{27}$ La organización se puso en funcionamiento a través de los exgestores de fondos de cobertura de los Estados Unidos [U.S. hedge fund managers] y persigue asegurar que sus propias contribuciones a la disminución de la pobreza sean tan efectivas como sea posible. Su página web es www.givewell.com, ultimo acceso 1 de diciembre de 2011. ASAP financió recientemente un evento de GiveWell en la Universidad de Yale, diseñado como escaparate público del trabajo de la organización y como medio de reclutar personal para la institución.

${ }^{28}$ Cfr. BANERJEE, A.V.: "“Making Aid Work," en COHEN, J. (ed.): Making Aid Work, Cambridge, MA, MIT Press, 2007, pp. 3-26; BANERJEE, A.V. - DUFLO, E.: Poor Economics: A Radical Rethink of the Way to Fight Global Poverty, New York, Public Affairs Books, 2011.
} 
Un proyecto [académico] adicional explora cómo las ventas de los recursos naturales de los gobernantes ilegales pueden ser cuestionadas empleando recursos legales existentes ${ }^{29}$.

Otra iniciativa [académica] está desarrollando una alternativa al modo en que las innovaciones académicas, actualmente, se incentivan y recompensan por medio de los márgenes de ganancias que dejan la protección de las patentes, poniendo estas medicinas fuera del alcance de los pobres del mundo ${ }^{30}$.

Estos son sólo algunos ejemplos de las diversas iniciativas de impacto directo a los que los académicos centrados en el tema de la pobreza pueden contribuir con su experiencia. De este modo se logran beneficios de escala al incrementar los efectos positivos y al amplificar su voz colectiva en relación a los aspectos clave de la pobreza.

La última categoría, la inclusión efectiva de la pobreza global en el diálogo sobre cómo y por qué mejorar las circunstancias de los desfavorecidos, es la menos desarrollada en el conjunto del mundo académico, si bien es también la más potencialmente significativa. La mencionada inclusión va más allá de los estudios empíricos de los contextos en que persiste la pobreza. Sin duda, se han conducido trabajos más rigurosos y agudos acerca de cómo los pobres más absolutos viven sus existencias en los últimos años por sociólogos, antropólogos, economistas y otros ${ }^{31}$. Trabajos análogos comenzaron a ser más sensibles a la pobreza global en relación a como ellos entienden sus propias privaciones y sobre los desafíos que encaran. Un ejemplo es el proyecto FemPov, que integra tres fases de entrevistas intensivas en dieciocho lugares de seis países ${ }^{32}$.

\footnotetext{
${ }^{29}$ Los detalles sobre esta iniciativa, promovida por el filósofo Leif Wenar del King's College London, se encuentra en www.cleantrade.org/.

${ }^{30}$ El desarrollo de esta iniciativa, the Health Impact Fund, se basa en los resultados de académicos y especialistas de todo el mundo (véase www.healthimpactfund.org, último acceso 1 de diciembre de 2011). Véase también BANERJEE, A. - HOLLIS, A. - POGGE, T.: "The Health Impact Fund: Incentives for Improving Access to Medicines" The Lancet 375 (2010), pp. 166-69.

${ }^{31}$ Para un ejemplo destacado que explora cómo aquellos que viven con menos de dos dólares diarios organizan sus ingresos y hacen frente al día a día, consúltese COLLINS, D. - MORDUCH, J. RUTHERFORS, S. - RUTHVEN, O.: Portfolios of the Poor: How the World's Poor Live on \$2 Per Day, Princeton, Princeton University Press, 2009.

32 Para más información, véase la página web del proyecto "Measuring Poverty and Gender Disparity" ["Midiendo la pobreza y las diferencias de género] en www.genderpovertymeasure.org/, último acceso 1 de diciembre de 2011.
} 
Un paso próximo destacado será introducir las voces de la pobreza global más directamente en los debates que nos preocupan tan profundamente. Algunos informes recientes, especialmente en la teoría política normativa, han realizado movimientos en esta dirección. Los teóricos han conducido entrevistas cualitativas con algunas personas que enfrentan privaciones graves. A partir de este trabajo, han sido capaces de presentar o hilvanar argumentos no sólo de activistas en nombre del pobre $^{33}$ sino también de inmigrantes ilegales, mujeres pobres y una minoría de grupos que sufren múltiples privaciones dentro de sus países $^{34}$. Tales informes, al incorporar los puntos de vista contextualizados de los pobres dentro de argumentaciones académicas sistematizadas sobre la pobreza global, completan y, además, movilizan mejor que el enfoque más directo $-\mathrm{y}$ muchas veces tremendamente potente, de las historias orales o las narraciones de hechos reales ${ }^{35}$.

Hay una gran variedad de posibilidades para promover la inclusión y permitir al pobre unirse al discurso global más directamente. La tecnología informática de conexión a través de video, por ejemplo, hace posible llevar en directo a las aulas de todo el mundo a activistas, a realizadores de documentales, representantes públicos y otros. Aunque hay que ser cauteloso a la hora de tomar a individuo como representante de la pobreza mundial, la tecnología permite fácilmente traer a

\footnotetext{
${ }^{33}$ Cfr. ACKERLY, B.: Universal Human Rights in a World of Difference, Cambridge, Cambridge University Press, 2008.

${ }^{34}$ Un informe que se centra en parte en los inmigrantes ilegales y en los pobres pertenecientes a las áreas de México y otras áreas desde las que inician su viaje, véase CABRERA, L.: The Practice of Global Citizenship. El estudio de campo de Cabrera incluye más de doscientas cincuenta entrevistas con inmigrantes, así como sobre los derechos de los inmigrantes y sobre los activistas antiinmigrantes en los Estados Unidos, México y la Europa Occidental. Un estudio que da pasos adelante incluyendo a las voces de las mujeres puede consultarse en NUSSMAUM, M.: Women and Human Development: The Capabilities Approach, Cambridge, Cambridge University Press, 2000. Jonathan Wolff, Avner De-Shalit y otros colegas entrevistaron a cien personas en Inglaterra e Israel, incluyendo a trabajadores sociales y a sus clients, para desarrollar una teoría normativa sobre aquellos que poseen menos basada en la teoría de las capacidades de Nussbaum Disadvantage, Oxford, Oxford University Press, 2007.

${ }^{35}$ Por ejemplo, las vulnerabilidades y privaciones sufridas por muchos Dalits son narradas por medio de sus propias voces en los dos volúmenes producidos por los grupos civiles de la India y financiados por los Países Bajos ALOYSIUS IRUDAYAM S.J. - MANGUBHAI, J.P. - LEE, J. G.: Dalit Women Speak Out: Violence Against Dalit Women in India, Delhi, National Campaign on Dalit Human Rights, 2006. Alicia Alarcón ofrece una crónica igual de enérgica en primera persona de las expericiencias de inmigrantes no autorizados en la frontera entre Estados Unidos y México en The Border Patrol Ate My Dust, Traduc. Ethriam Cash Brammer de Gonzales, Houston, Arte Publico
} 
organizaciones de base procedentes de las regiones más pobres, así como a individuos corrientes. Esto se puede trasladar a diálogos en las aulas, a ciertos tipos de encuentros académicos y a otros escenarios.

La pretensión general consiste en hacer posible que los pobres del mundo compartan su propia "perspectiva privilegiada" sobre sus vidas. Esta frase está tomada de tratados de gobiernos democráticos, donde se asume como una razón fundamental para una toma de decisiones inclusiva ${ }^{36}$. Ni siquiera los gobernantes más benévolos podrán tener jamás una información completa sobre cómo pueden afectar sus decisiones y políticas a los individuos; así pues, es importante permitir a todos que den su opinión. De la misma forma, el discurso y los proyectos relativos a la pobreza global se pueden robustecer a través de las opiniones de aquellos que, de facto, sufren una pobreza grave y las de aquellos un poco más ricos que comparten el mismo contexto y están dispuestos a defender los intereses de los más necesitados. Un ejemplo a subrayar de este último punto sería la Campaña Nacional de los Derechos Humanos de los Dalit, afincada en Delhi. Sus miembros son fundamentalmente los Dalit (casta intocable) y trabajan en nombre de millones de Dalits en la India, que continúan sufriendo algunas de las condiciones sociales y materiales más adversas del planeta.

\section{Posibles objeciones}

No estamos pidiendo que una legión de grandes "planificadores",37 descienda de sus torres de marfil y erradiquen la pobreza global de un plumazo. Más bien, esperamos que muchos más académicos se animen a compartir sus conocimeintos de expertos y sus visiones prácticas sobre la pobreza y el compromiso público entre ellos, con el fin de hacer más

\footnotetext{
${ }^{36}$ Cfr. SHAPIRO, I.: The State of Democratic Theory, Princeton, Princeton University Press, 2003, pp. 39-43.

${ }^{37}$ El concepto es de William Easterly. Él establece un profundo contraste entre los esfuerzos realizados por los "Planners", que desean aplicar diseños de amplio espectro y centralizados a los problemas sociales globales y los "Searches", que, trabajan para conseguir soluciones específicas para problemas concretos (cfr. EASTERLY: The White Man's Burden: Why the West's Efforts to Aid the Rest Have Done So Much Ill and So Little Good, Oxford, Oxford University Press, 2006, especialmente la parte I.
} 
efectivas las contribuciones de la academia en relación a la disminución de la pobreza. Un grupo como ASAP puede contribuir a asegurar que las mejores ideas se canalicen en el discurso público. Además, es capaz de promover un diálogo e mayor interacción entre las disciplinas. Por ejemplo, como Keith Horton ha aseverado ${ }^{38}$, se puede obtener enorme beneficio de animar a un diálogo más robusto entre los teóricos normativos centrados en una distribución más justa del producto social global y entre los académicos empíricos dedicados a la ayuda y el desarrollo. Este diálogo interdisciplinar fomenta que los académicos sean conscientes de los mejores descubrimientos disciplinares o de los argumentos procedentes de los diversos subcampos. Así, ayudarán a evitar repetir los viejos caminos ya andados o aparecer como los "ingenuos bienhechores" referidos arriba ${ }^{39}$. Los representantes de las ONG pueden proporcionar, además, ideas valiosas sobre las iniciativas, éxitos y fracasos del pasado, especialmente sobre los puntos a acentuar en el desarrollo y la distribución de la ayuda ${ }^{40}$. Un diálogo más sistemático traerá a la palestra complementariedades entre la academia y las iniciativas de las ONGs. Aún más, dotará de un medio para airear y resolver auténticas diferencias.

Tales diferencias son el núcleo de una objeción ofrecida por Gerald Gaus ${ }^{41}$. Él afirma que los filósofos no deberían intentar "aplicar" la ética al discurso público. Esta acción desalienta el equilibrio imparcial de las diversas razones y alienta una polémica retórica que sólo pretende alistar partidarios. La misma objeción general se podría aplicar a la presentación de lo datos empíricos. Se podría argumentar que una vez el economista o el especialista en desarrollo dan el salto a la arena pública, los asuntos relativos a la recopilación de datos y a la honestidad sobre las conclusiones se pierden fácilmente en el camino que conduce a conseguir ciertos resultados políticos. En lugar de tomar partido, haríamos mejor en seguir los argumentos y evidencias a donde nos lleven y a reconocer que,

\footnotetext{
${ }^{38}$ Cfr. HORTON, K.: “An Appeal to Aid Specialists,” Development Policy Review 28, no 1 (2010), pp. 27-42.

${ }^{39}$ Véanse las contribuciones de Roger Riddell sobre este asunto.

${ }^{40}$ Véanse las contribuciones de Martin Kirk sobre este asunto.

${ }^{41}$ Cfr. GAUS, G.: "Should Philosophers Apply Ethics?", op.cit.
} 
en general, en los casos complicados existe un "pluralismo razonable" de puntos de vista que compiten entre sí.

Nosotros obtuvimos la conclusión contraria respecto a la cuestión del compromiso. Reafirmando la perspectiva bosquejada arriba, nosotros sostenemos que precisamente porque los académicos son los mejor situados para examinar evidencias y argumentos con rigor al tratar de derivar los juicios mejor fundamentados posibles, por eso mismo deben implicarse plenamente en los debates sobre la pobreza global. Los desacuerdos sobre los temas relevantes persistirán, sin duda. Sería ingenuo esperar un acuerdo completo de todos los académicos sobre, por ejemplo, los criterios que reemplazarán al ODM (sus indicadores de pobreza, criterios de medida, medios de aplicación, etc). Sería igualmente desacuerdo conduce inevitablemente a un callejón sin salida y que resulta imposible identificar los puntos de acuerdo subyacentes, a través del diálogo entre especialistas.

Es completamente plausible pensar que ese acuerdo se puede alcanzar sobre algunos aspectos importantes conectados al proyecto de reemplazo [de los ODM], por ejemplo, someter a los estados más ricos a obligaciones más específicas ${ }^{42}$. En cualquier caso, en el mundo real, habrá un resultado: algún compromiso nuevo o ninguno. Si se adoptan nuevas metas, estas podrían ser nuevamente inespecíficas en relación a los destinatarios, dando libertad a todos para celebrar que se han conseguido puesto que no se singulariza quién ha de realizarlas. Sin embargo, por otro lado, se pueden proponer nuevas metas que incluyan una división de tareas realista que requiera agentes específicos y efectivos capacitados para cada acción, que den cuenta de un calendario bien definido. Observando cuánto está en juego en relación a los resultados, los académicos interesados en el tema de la pobreza tienen buenas fuertes razones para desear implicarse en los debates. Trabajar en el seno de un grupo como ASAP nos ayuda a trascender el cúmulo de estrechos desacuerdos, a ponernos en camino hacia la identificación de las asunciones y conclusiones ampliamente compartidas y a desarrollar

\footnotetext{
${ }^{42}$ Véase también HORTON, K.: “Academics Stand Against Poverty: The Story So Far" (2011). Disponible online en http://academicsstand.org/article/academics-stand-against-poverty-the-story-sofar/, último acceso 1 de diciembre de 2011.
} 
aquellos caminos más significativos para el público y para los legisladores.

Otra objeción se alza en torno a las exigencias [que descansan sobre los hombros de los académicos]. Es injusto, aseverarán algunos, esperar que los académicos, que ya tienen muchas demandas de tiempo y energía, den de sí mismos a la pobreza global más que otras personas en mejores condiciones que ellos. Una posible respuesta a esta objeción señala que no se pide a los académicos que hagan más que otros, sino sencillamente que sus acciones posean más alcance. Así pues, si se pide a las personas con una seguridad material que donen un diez por ciento de su tiempo, no se solicita a los investigadores y profesores más de ese tiempo. Sin embargo, al poseer un mayor nivel de pericia y conocimientos, sus resultados conseguirían mayor repercusión que los de quienes no pertenecen a este entorno (aunque ambos deberían conseguir tanto como fuera posible con el tiempo y los recursos a su mano para la erradicación de la pobreza).

Esta propuesta puede interpretarse como muy conservadora. Alguien que con cada hora de su tiempo consiguiese un aumento de los ingresos de la gente más desfavorecida de cien dólares, debería ofrecer más horas que aquellos sujetos no expertos que sólo obtuviesen un aumento de diez dólares por cada hora de su tiempo. ¿Acarrea esta perspectiva lastres excesivos e injustos para los académicos? Una organización como ASAP ayudará a evitar esto. Al jugar el rol de coordinación, ASAP es capaz de incrementar el número de académicos que contribuyan y organizar su colaboración de forma que sus cargas sean minimizadas al aumentar la eficiencia y la distribución equitativa ${ }^{43}$. Compartiendo información y a través del diálogo, los miembros de ASAP pueden ayudarse entre ellos por medio de la conexión y ampliación del impacto de sus esfuerzos, puesto que será posible abrir si experiencia y energía a usos más eficaces.

Una objeción final se funda en los deberes recíprocos hacia los compatriotas. Se podría argüir que, si los académicos deben asumir un rol público en la lucha contra la pobreza, deberían priorizar la pobreza de la propia casa. Tenemos deberes de reciprocidad hacia los compatriotas, que

${ }^{43}$ Cfr. SHUE, H.: “Mediating Duties,” Ethics 98, no 4 (1988), pp. 687-704. 
han contribuido en mayor cuantía a costear el tiempo que dedicamos a nuestras propias agendas y a diseminar nuestras ideas. En ese sentido, deberíamos trabajar en primer lugar a aliviar la pobreza doméstica relativa más que la pobreza absoluta global.

A pesar de ello, esta objeción se mantiene en pie sólo si los deberes de reciprocidad triunfan sobre otros deberes; sin embargo, esta premisa es cuestionable. Se puede defender que las instituciones supranacionales que se mantienen gracias a nuestra cooperación son gravemente injustas a la vista de la ingente pobreza evitable que generan. Deberíamos poner fin a esta injusticia o proteger a sus víctimas en virtud de un deber negativo (no causar daño) que es más relevante que nuestros deberes hacia los compatriotas $^{44}$. Se puede también afirmar que nuestros deberes generales positivos hacia las personas que viven en pobreza extrema en el extranjero son más urgentes porque sus necesidades son mayores y es más barato satisfacerlas.

Se puede suscitar una cuestión más fundamental en relación a la justicia de las condiciones del trasfondo de las que se dicen que generan los deberes de reciprocidad. Hasta que se demuestre que las exclusiones y las restricciones territoriales asociadas al sistema global actual sean moralmente defendibles, un sistema de reciprocidad erigido sobre ellas está abierto a ser cuestionado. Para ilustrar este punto, imagine el dueño de un esclavo que lo entrega a otro propietario con la condición de que, más tarde, recibirá un esclavo similar a cambio. Ese contrato recíproco no ayuda a justificar el sistema de esclavitud sobre el que descansa. Análogamente, el hecho de sentir una necesidad de devolver los favores de los compatriotas dentro de una sociedad rica o de compensarlos por la imposición de leyes que limitan nuestra libertad ${ }^{45}$, no pueden sostenerse como la prueba de que los deberes domésticos de reciprocidad triunfan sobre los de la pobreza global, a menos de que se pueda establecer que el sistema sobre el descansan los deberes domésticos es justo. Si la riqueza de la sociedad se sostiene sobre un sistema global de normas que mantiene, de forma evitable, a miles de millones de personas bajo condiciones de pobreza, entonces la prioridad de sus miembros de

\footnotetext{
${ }^{44}$ Cfr. POGGE, T.: World Poverty and Human Rights, especialmente los capítulos 3-5.

${ }^{45}$ Cfr. MILLER, R.: "Cosmopolitan Respect and Patriotic Concern," Philosophy and Public Affairs, $\mathrm{n}^{\mathrm{o}} 27$ (1998), pp. 202-24.
} 
realizar donaciones entre ellos podría ser una violación de los derechos humanos antes que el cumplimiento de un contrato moralmente válido de reciprocidad. Estos asuntos relativos a la urgencia relativa de nuestros deberes son complejos y académicos formados pueden realizar contribuciones importantes para clarificarlos y resolverlos ${ }^{46}$.

\section{Conclusión}

Cerraremos nuestro trabajo invitando a académicos, a estudiantes graduados y licenciados interesados en la disminución de la pobreza a unirse a Académicos contra la pobreza [Academics Stand Against Poverty] (www.academicsstand.org). También son bienvenidos los miembros afiliados procedentes de ONGs interesadas en la pobreza y todos aquellos que se dedican al servicio público. Será de utilidad aquí explicar algo más de las bases de ASAP.

La iniciativa se puso en marcha por académicos de Australia y Estados Unidos que buscaban formas de mejorar el conocimiento académico sobre la pobreza global y promover la colaboración entre las disciplinas ${ }^{47}$. La organización inicial condujo a la realización de reuniones formales para el lanzamiento en 2010-2011 que movilizaron a numerosos participantes en las Universidades de Yale, Birmingham, Oslo, la University of Notre Dame London Center y la Universidad de Delhi. Los participantes de cada encuentro contribuyeron a identificar prioridades para la organización y proporcionaron feedback a otros proyectos propuestos.

Otros esfuerzos iniciales se centraron en el desarrollo de la web, que pretende ofrecer información sobre el impacto y ampliación global de los proyectos; asimismo, permite a los académicos con ideas similares ponerse en contacto y colaborar entre ellos de diferentes formas. Entre los proyectos informativos se incluye compilar una base de datos extensa de

\footnotetext{
${ }^{46}$ Véanse estudios recientes ofreciendo críticas de la prioridad a la distribución entre los compatriotas en POGGE, T.: World Poverty and Human Rights; CANEY, S.: Justice Beyond Borders: A Global Political Theory, Oxford, Oxford University Press, 2005; MOELLENDORF, D.: Global Inequality Matters, Basingstoke, Palgrave Macmillan, 2009; BROCK, G. Global Justice: A Cosmopolitan Account, Oxford, Oxford University Press, 2009.

${ }^{47}$ Cfr. HORTON, H. “Academics Stand Against Poverty: The Story So Far”, op.cit.
} 
centros universitarios, institutos e iniciativas focalizadas en la pobreza global, así como proveer de proyectos de impacto y campañas de las ONG en las que los académicos puedan contribuir. Se pretende que la base de datos proporcione un lugar para que los académicos entren en contacto con ideas que ya están en funcionamiento y para que encuentren modelos e inspiración para proyectos independientes. Una red paralela de la web permite a grupos externos, incluidas asociaciones y ONGs, colaborar con los miembros online de ASAP.

La web de ASAP ofrecerá, además, muchas oportunidades para que los académicos compartan información y se comprometan directamente en el diálogo a través de la sección Foro "Pobreza mundial". Este foro anexará pequeños artículos que destaquen las recientes investigaciones focalizadas en eventos claves de la política global tales como aquellos relacionados con el reemplazo de los ODM. Por otra parte, ofrecerá recursos sobre ciertos medios efectivos de alcanzar las audiencias más amplias y a los legisladores. Los usuarios individuales podrán ofrecer feedback online para anunciar "lecturas recomendadas" y formular cuestiones abiertas al diálogo común.

ASAP también pretende alimentar y ofrecer una plataforma colaborativa para algunos proyectos de impacto. Uno relevante de los más recientes, ya mencionado, es el proyecto de Consenso para la Pobreza Mundial, que, inicialmente, desea reunir las contribuciones de los académicos al diálogo de la sustitución de los ODM y que identificará los puntos comunes de acuerdo que podrán tomar parte de modo significativo en los aspectos de política global. Se están desarrollando proyectos adicionales de impacto, es más, ASAP se dedica a ayudar a colaboradores potenciales de proyectos a encontrarse entre sí y a facilitar información compartida sobre modos de financiación y de apoyo.

Comenzamos este ensayo en un modo ciertamente pesimista, subrayando los caminos que buscaban la erradicación de la pobreza y que que no habían gozado de suficiente impacto. Existen varias razones para ello. Una es el exceso de "buenas ideas". En este sentido, hemos de poner el acento en el Foro Mundial Social [World Social Forum], donde miles de personas presentaron miles de buenas ideas, obligadas a pisarse entre sí. ASAP puede ayudar a superar este problema seleccionando y extendiendo las mejores ideas y centralizando los esfuerzos de muchos en 
su realización. Resulta difícil saber por adelantado cuantos académicos colaboran a través de las naciones y las disciplinas que pueden unirse a la lucha contra la pobreza. Pero los roles especiales que jugamos en las sociedad y nuestras capacidades nos dan razones para creen que poder realizar contribuciones sustanciales y diferenciadas del resto. La necesidad de una acción más efectiva es, sin duda, urgente. Hagamos un esfuerzo más coordinado como investigadores y profesores para ayudar a construir alianzas de gentes y de organizaciones que luchan por poner fin a la pobreza severa mundial. 


\section{RESEÑAS BIBLIOGRÁFICAS / REVIEWS}


